\title{
Manual Therapy
}

National Cancer Institute

\section{Source}

National Cancer Institute. Manual Therapy. NCI Thesaurus. Code C153321.

A physical treatment primarily used to treat musculoskeletal pain and disability through physical manipulation of the body. 